\title{
Search for production of an invisible dark photon in $\pi^{0}$ decays
}

\section{The NA62 collaboration}

\section{E-mail: tommaso.spadaro@cern.ch, marco.mirra@cern.ch}

ABstRACT: The results of a search for $\pi^{0}$ decays to a photon and an invisible massive dark photon at the NA62 experiment at the CERN SPS are reported. From a total of $4.12 \times 10^{8}$ tagged $\pi^{0}$ mesons, no signal is observed. Assuming a kinetic-mixing interaction, limits are set on the dark photon coupling to the ordinary photon as a function of the dark photon mass, improving on previous searches in the mass range $60-110 \mathrm{MeV} / c^{2}$. The present results are interpreted in terms of an upper limit of the branching ratio of the electro-weak decay $\pi^{0} \rightarrow \gamma \nu \bar{\nu}$, improving the current limit by more than three orders of magnitude.

KEywords: Beyond Standard Model, Exotics, Fixed target experiments, Rare decay

ARXIV EPRINT: 1903.08767 


\section{Contents}

1 Introduction 1

2 Beam line and detector $\quad 2$

3 Analysis principle $\quad 4$

3.1 Selection of the normalization sample 5

$\begin{array}{ll}3.2 & \text { Selection of the signal sample }\end{array}$

$\begin{array}{lll}3.3 & \text { Background evaluation } & 7\end{array}$

4 Search for an $A^{\prime}$ signal $\quad 8$

4.1 Efficiency corrections 9

$\begin{array}{ll}4.2 \text { Evaluation of the upper limit } & 10\end{array}$

$\begin{array}{lll}4.3 & \text { Systematic errors } & 11\end{array}$

$5 \quad$ Search for the $\pi^{0} \rightarrow \gamma \nu \bar{\nu}$ decay 12

6 Conclusions 12

$\begin{array}{ll}\text { The NA62 collaboration } & 16\end{array}$

\section{Introduction}

One of the possible extensions of the Standard Model (SM) aimed at explaining the abundance of dark matter in our universe predicts a new $\mathrm{U}(1)$ gauge-symmetry sector with a vector mediator field $A^{\prime}$, often called "dark photon". In a simple realization of such a scenario [1,2], an $A^{\prime}$ field $A_{\mu \nu}^{\prime}$ with mass $M_{A^{\prime}}$ interacts with the SM photon through a kinetic-mixing Lagrangian,

$$
\epsilon A_{\mu \nu}^{\prime} F^{\mu \nu}
$$

where $F_{\mu \nu}$ represents the electromagnetic field tensor and $\epsilon \ll 1$ is the coupling constant. A consequence of this interaction is the transition $\pi^{0} \rightarrow A^{\prime} \gamma$ with branching ratio, BR:

$$
\mathrm{BR}\left(\pi^{0} \rightarrow A^{\prime} \gamma\right)=2 \epsilon^{2}\left(1-\frac{M_{A^{\prime}}^{2}}{M_{\pi^{0}}^{2}}\right)^{3} \times \mathrm{BR}\left(\pi^{0} \rightarrow \gamma \gamma\right)
$$

In a general picture, the above Lagrangian might be accompanied by further interactions, both with SM matter fields and with a secluded hidden sector of possible dark-matter candidate fields. If these are lighter than the $A^{\prime}$, the dark photon would decay mostly invisibly. 


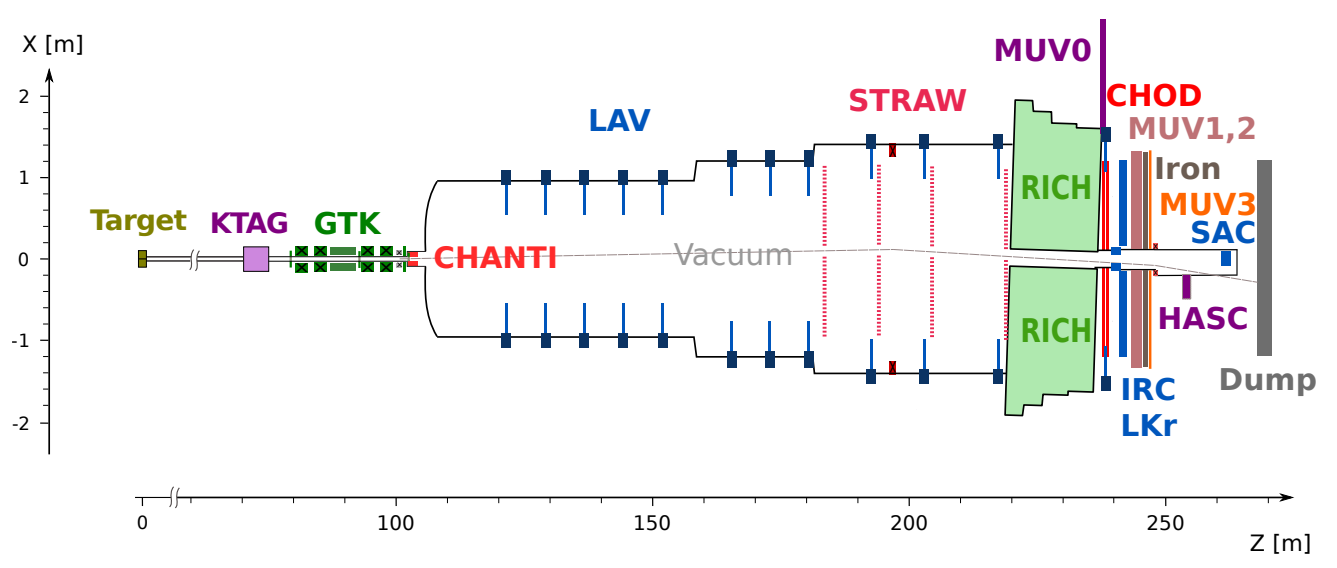

Figure 1. Schematic top view of the NA62 beam line and detector. The trajectory of a beam particle is shown, travelling in vacuum and crossing the detector apertures. A dipole magnet between the MUV3 and SAC systems deflects beam particles out of the SAC acceptance.

The search for an invisible $A^{\prime}$ is performed with a missing-mass technique from the full reconstruction of the decay chain

$$
K^{+} \rightarrow \pi^{+} \pi^{0}, \quad \pi^{0} \rightarrow A^{\prime} \gamma
$$

An abundant flux of $K^{+}$mesons is provided by a high-energy unseparated hadron beam from the CERN Super Proton Synchrotron (SPS). The search is performed using the NA62 experiment, which has the main goal of measuring the BR of the rare decay $K^{+} \rightarrow \pi^{+} \nu \bar{\nu}$ with $10 \%$ precision. The design of the experiment guarantees high intensity, full particle identification, hermetic coverage, low material budget and high-rate tracking. The NA62 detector has been fully operational since 2016. The results from the analysis of a subsample of 2016 data are reported, corresponding to $1 \%$ of the statistics collected by NA62 in 2016-2018.

\section{Beam line and detector}

The beam line and detector, shown schematically in figure 1, are described in detail elsewhere [3]. Here, the aspects relevant to the search for the decay chain described in eq. (1.3) are outlined.

A proton beam of $400 \mathrm{GeV} / c$ in a $4.8 \mathrm{~s}$ long spill from the SPS hits a beryllium target to produce an intense $75 \mathrm{GeV} / c$ secondary beam of positive particles, $6 \%$ of which are charged kaons. The secondary beam is selected with a $1 \%$ RMS momentum bite and is transported to the decay region more than $100 \mathrm{~m}$ downstream of the target. For the present measurement, the typical beam particle rate at the entrance of the decay volume is $300 \mathrm{MHz}$. Incoming kaons are positively identified by a differential Cherenkov counter read out by photomultipliers (PMs) grouped into eight sectors (KTAG): requiring a set of in-time signals (KTAG candidate) in five or more sectors identifies a $K^{+}$with 70 ps time resolution. 
A magnetic spectrometer hosting three stations of Si-pixel detectors (GTK) reconstructs tracks for individual beam particles with $100 \mathrm{ps}$ resolution and provides the longitudinal momentum and direction with $0.15 \mathrm{GeV} / c$ and $16 \mu \mathrm{rad}$ resolutions, respectively.

For the present analysis, kaon decays in a $50 \mathrm{~m}$ long fiducial volume are reconstructed. This volume is contained in a decay tank evacuated to $10^{-6}$ mbar. The momentum and position of the daughter particles are measured by a spectrometer consisting of two strawtube chambers (STRAW) on either side of a dipole magnet providing a transverse horizontal momentum kick of $270 \mathrm{MeV} / c$. Reconstructed STRAW tracks measure the momentum with a resolution $\sigma_{p} / p$ in the range of $0.3-0.4 \%$. Daughter photons are detected by a hermetic system involving two lead-scintillator calorimeters (IRC and SAC) for emission angles with respect to the $Z$ axis $\theta<1 \mathrm{mrad}$, a liquid krypton electromagnetic calorimeter (LKr) for $1<\theta<10 \mathrm{mrad}$, and a system of 12 annular lead-glass detectors (LAV) for $10<\theta<50 \mathrm{mrad}$. The detection inefficiency is below $10^{-3}$ for photons directed towards the IRC and SAC calorimeters with energy above $6 \mathrm{GeV}$; below $10^{-5}$ for photons hitting the LKr calorimeter with energy above $10 \mathrm{GeV}$; below $10^{-3}$ for photons hitting the LAV detector with energy above $1 \mathrm{GeV}$. A localized set of LKr cells with coincident signals is grouped into a cluster, providing measurements of energy, transverse coordinates, and time with resolutions of $\sigma_{E} / E=4.8 \% / \sqrt{E[\mathrm{GeV}]} \oplus 11 \% / E[\mathrm{GeV}] \oplus 0.9 \%, 1 \mathrm{~mm}$, and between 0.5 and 1 ns depending on the amount and type of energy deposition, respectively.

A ring-imaging Cherenkov detector $(\mathrm{RICH})$, with 70 ps resolution, identifies secondary charged pions. Two downstream scintillator hodoscopes provide fast time response for charged particles: the CHOD, a matrix of tiles read out by SiPMs, has a time resolution below $1 \mathrm{~ns}$; the NA48-CHOD, composed of two orthogonal planes of scintillator slabs, has 200 ps resolution for coincidence between vertical and horizontal slabs (NA48-CHOD candidate). Two hadronic iron/scintillator-strip sampling calorimeters (MUV1,2) and an array of scintillator tiles located behind $80 \mathrm{~cm}$ of iron (MUV3, with $400 \mathrm{ps}$ time resolution) supplement the pion/muon identification system. The overall probability for identifying a $\mu^{+}$as a $\pi^{+}$in the momentum range $15-35 \mathrm{GeV} / c$ is at the level of $10^{-7}$ [4].

Information from the NA48-CHOD, CHOD, RICH, MUV3, LKr, and the most downstream LAV station (LAV12) is hardware-processed to issue level-zero (L0) trigger signals with a frequency up to $1 \mathrm{MHz}$. The L0 trigger condition used to search for the decay chain of eq. (1.3), denoted as signal trigger, aims to select final states with one emitted $\pi^{+}$and missing energy. It requires a signal in the RICH in coincidence within $10 \mathrm{~ns}$ with a signal in at least one CHOD tile. No signals in opposite CHOD quadrants must be found within the $10 \mathrm{~ns}$ window, thus reducing the contribution of $K^{+} \rightarrow \pi^{+} \pi^{+} \pi^{-}$decays and in general of final states with multiple charged particles; this condition is called $Q X$-veto in the following. No signals in the MUV3 detector must be present, thus reducing the contribution of $K^{+} \rightarrow\left(\pi^{0}\right) \mu^{+} \nu$ decays. No more than one in-time signal must be found in LAV12 and no more than $20 \mathrm{GeV}$ of total energy deposit in time in the LKr calorimeter must be reconstructed. These conditions reduce the contribution of multi-photon final states and are particularly effective in rejecting forward-emitted photons from $\pi^{0}$ decays.

A software trigger (L1) reconstructs data from the KTAG, LAV and STRAW detectors to further enforce the presence of a charged kaon and to reject final states with additional 
particles emitted at large angle. The charged kaon must be positively identified using KTAG information within $10 \mathrm{~ns}$ of the L0 trigger RICH-based time. At least one STRAW track must be reconstructed, corresponding to a particle with momentum below $50 \mathrm{GeV} / c$ and a point of closest approach (less than $20 \mathrm{~cm}$ ) to the nominal beam axis upstream of the first STRAW chamber. Events with in-time signals in three or more LAV blocks are rejected. These conditions reduce the trigger rate by a factor of 100 .

For normalization, the analysis uses data taken with a concurrent minimum-bias L0 trigger ("control trigger") based on NA48-CHOD information. The control trigger requires one or more time coincidences between horizontal and vertical planes of scintillators in the NA48-CHOD hodoscope, and is downscaled by a factor of 400 .

\section{Analysis principle}

Assuming a dominant invisible decay of the $A^{\prime}$ (or a long-lived $A^{\prime}$ producing no observable interaction in the LKr calorimeter), the experimental signature for the events described in eq. (1.3) is given by a kaon decaying into a charged pion and a photon hitting the LKr calorimeter, with missing energy and momentum. The kaon and pion momenta are measured with the GTK and STRAW detectors, respectively, and the corresponding 4momenta are denoted $P_{K}$ and $P_{\pi}$. The measurement of the position of impact and the energy released in the LKr allow the determination of the photon 4-momentum $P_{\gamma}$, assuming emission from the decay vertex. The squared missing mass

$$
M_{\text {miss }}^{2}=\left(P_{K}-P_{\pi}-P_{\gamma}\right)^{2}
$$

is expected to peak at $M_{A^{\prime}}^{2}$ for the decay chain in eq. (1.3) and at zero for the most abundant background, $\pi^{0} \rightarrow \gamma \gamma$ with one photon undetected.

A high-purity kinematic identification of the $K^{+} \rightarrow \pi^{+} \pi^{0}$ decays is performed by reconstructing solely the $K^{+}$and $\pi^{+}$particles. The number of $K^{+} \rightarrow \pi^{+} \pi^{0}$ decays, $n_{\pi^{0}}$, counted in the control-trigger sample defines the statistics of tagged $\pi^{0}$ mesons used for normalization.

Additional conditions are required for signal-triggered events, in order to enforce the sole presence of a $\pi^{+}$and one photon in the final state. The selection efficiency for these additional requirements and the signal-trigger efficiency depend on $M_{A^{\prime}}$ and are denoted as $\varepsilon_{\text {sel }}$ and $\varepsilon_{\text {trg. }}$. A peak search in the positive tail of the $M_{\text {miss }}^{2}$ background distribution is performed by comparing the number of events in a sliding $M_{\text {miss }}^{2}$ window to the background expectation. For illustration, the distributions of $M_{\text {miss }}^{2}$ from a Monte Carlo (MC) simulation of the NA62 apparatus when injecting $A^{\prime}$ signals with masses of 60,90 , and $120 \mathrm{MeV} / c^{2}$ and a coupling strength $\epsilon^{2}=2.5 \times 10^{-4}$ (see eq. (1.2)) are shown in figure 2 . These are superimposed on the expected contribution from a control-trigger data sample with fully reconstructed $\pi^{0} \rightarrow \gamma \gamma$ in which one of the two photon LKr clusters, randomly chosen, is artificially excluded. The data distribution is scaled to $n_{\pi^{0}}$. Each MC distribution is scaled to the equivalent number of tagged $\pi^{0}$ mesons corresponding to the generated statistics.

The estimate of the number of signal events $n_{\text {sig }}$ in a given $M_{\text {miss }}^{2}$ window is normalized to the number $n_{\pi^{0}}$ to yield the BR for the decay $\pi^{0} \rightarrow A^{\prime} \gamma$ (and hence the $\epsilon^{2}$ coupling 


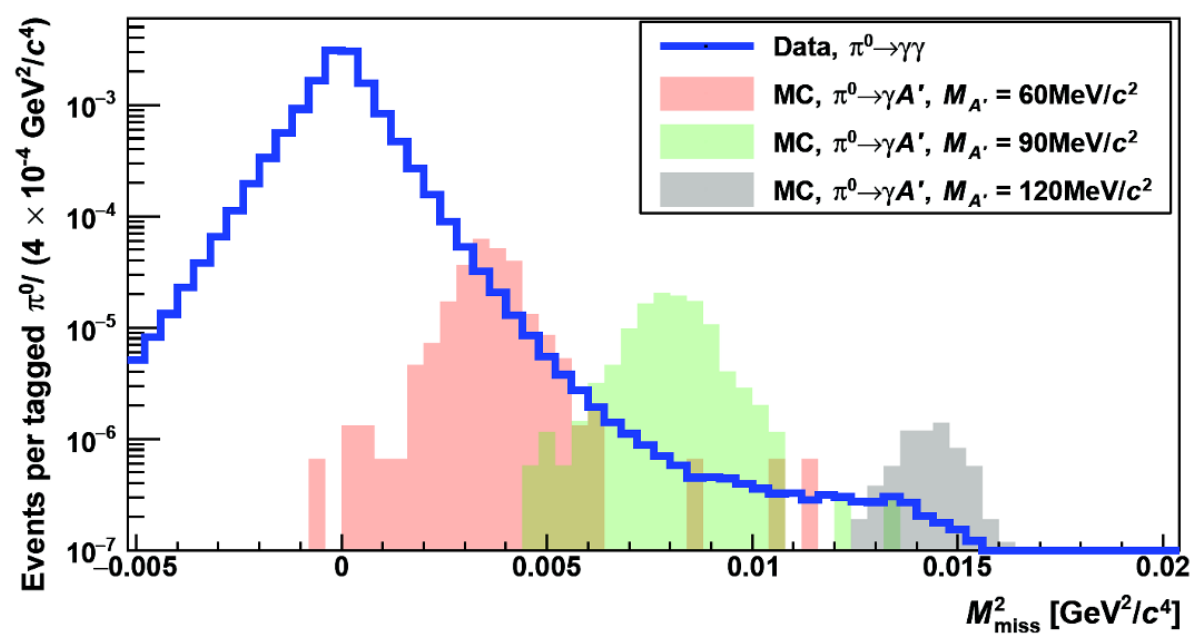

Figure 2. Distributions of the squared missing mass evaluated from $K^{+}$decays with one photon and one $\pi^{+}$reconstructed (eq. (3.1)). Data from $\pi^{0} \rightarrow \gamma \gamma$ with one photon, randomly chosen, assumed to be undetected are shown by the blue line. The expected spectra from MC simulations of $\pi^{0} \rightarrow A^{\prime} \gamma$ with a coupling strength $\epsilon^{2}=2.5 \times 10^{-4}$ and $A^{\prime}$ masses of 60 (red), 90 (green) and $120 \mathrm{MeV} / c^{2}$ (grey) are also shown. For details about the normalization used, see text.

strength):

$$
\mathrm{BR}\left(\pi^{0} \rightarrow A^{\prime} \gamma\right)=\mathrm{BR}\left(\pi^{0} \rightarrow \gamma \gamma\right) \frac{n_{\mathrm{sig}}}{n_{\pi^{0}}} \frac{1}{\varepsilon_{\mathrm{sel}} \varepsilon_{\text {trg }} \varepsilon_{\text {mass }}},
$$

where the correction factor $\varepsilon_{\text {mass }}$ accounts for the acceptance of the sliding $M_{\text {miss }}^{2}$ window used. The geometrical acceptance and the $\pi^{0}$-tagging efficiency are identical for the signal and normalization channels and therefore cancel exactly in eq. (3.2). Part of the sample is solely used for a data-driven background evaluation, reducing the size of the dataset exploited in the signal search.

\subsection{Selection of the normalization sample}

The normalization sample is selected as follows.

- Events with one charged daughter particle are required: exactly one STRAW goodquality track geometrically associated with a NA48-CHOD candidate must be reconstructed. The STRAW track is the $\pi^{+}$candidate in the decay $K^{+} \rightarrow \pi^{+} \pi^{0}$. The track momentum must lie in the range $15<p_{\pi}<35 \mathrm{GeV} / c$, ensuring at least $40 \mathrm{GeV}$ of missing energy for a nominal kaon momentum of $75 \mathrm{GeV} / c$.

- To achieve high-purity pion identification, the $\pi^{+}$-candidate track is associated in time with a single ring from the RICH consistent with the track direction. The NA48CHOD hodoscope is used as the time reference for the association to the RICH, which is then used as a reference for all subsequent associations. The track must be geometrically associated with in-time energy deposits from the LKr, MUV1, and MUV2 calorimeters. No in-time MUV3 signal must be geometrically associated with the 
track. Information from the LKr, MUV1, and MUV2 is combined in a multivariate classifier leading to a $\mu$-to- $\pi$ mis-identification probability of $10^{-7}$ [4].

- The $\pi^{+}$-candidate track must be associated in space and time with exactly one beam track reconstructed with the GTK detector. Tight requirements are applied for this association to minimize the kinematic tails in the reconstruction: the matching time difference must be less than $400 \mathrm{ps}$ and the spatial distance of minimum approach cannot exceed $5 \mathrm{~mm}$. The point of closest approach of the $\pi^{+}$-candidate and beam tracks is taken as the reconstructed decay vertex. Its longitudinal position must lie in the interval $115<Z<165 \mathrm{~m}$ (figure 1).

- The beam particle is identified as a charged kaon by its association in time with a KTAG candidate with signals in five or more sectors. The kaon-candidate momentum reconstructed by the GTK spectrometer must lie in the range $72<p_{K}<78 \mathrm{GeV} / c$, to be consistent with the beam momentum.

- The squared missing mass is required to be consistent with the squared $\pi^{0}$ mass: $0.013<\left(P_{K}-P_{\pi}\right)^{2}<0.023 \mathrm{GeV}^{2} / c^{4}$.

These conditions select $K^{+} \rightarrow \pi^{+} \pi^{0}(\gamma)$ decays (inclusive of the inner-bremsstrahlung radiative component, IB) with contamination below the per-mil level. The total number of selected events is 1030155 . After accounting for the control-trigger downscaling factor of 400 , the number of tagged $\pi^{0}$ mesons corresponding to the signal trigger sample is $n_{\pi^{0}}=4.12 \times 10^{8}$. It has been checked that the statistical error on the downscaling has a negligible impact.

\subsection{Selection of the signal sample}

The algorithm described in the previous section is also applied to signal-triggered events. Further requirements are applied to identify the decay chain of eq. (1.3).

- No in-time signals from the LAV and SAC-IRC systems must be present.

- Exactly one in-time LKr cluster with energy $E_{\gamma}>1.5 \mathrm{GeV}$ is required at least $20 \mathrm{~cm}$ away from the pion impact point. The selected LKr cluster is assumed to be due to a photon originating from the decay vertex: its energy and position are used to evaluate the photon momentum. The missing momentum $\vec{p}_{\text {miss }}$ evaluated from the kaon, pion, and photon momenta must extrapolate from the decay vertex to the LKr calorimeter and any activity in the LKr around the $p_{\text {miss }}$ impact point must not have a total energy in excess of $1 \mathrm{GeV}$. These conditions ensure further rejection against additional photons. The impact point of $\vec{p}_{\text {miss }}$ must be at least $20 \mathrm{~cm}$ away from the LKr clusters associated with the pion and photon, thus minimizing energy sharing (isolation cut).

- No in-time RICH signals may be found apart from those reconstructing the pion Cherenkov ring, thus minimizing the contribution from upstream photon conversions in the STRAW chamber and RICH vessel materials. 
- A reconstruction bias may occur when a photon converts before reaching the LKr sensitive volume: if one particle of the $e^{+} e^{-}$pair from the conversion is undetected, the energy of the reconstructed photon cluster tends to be underestimated, occasionally by several $\mathrm{GeV}$. This effect has an impact on the background due to $\pi^{0} \rightarrow \gamma \gamma$ decays with one photon lost. The energy of the undetected photon is usually below $1 \mathrm{GeV}$, therefore a bias in the reconstruction of the detected photon may induce a correlated shift of the missing energy and of $M_{\text {miss }}^{2}$ towards positive values. Moreover, events with a systematic underestimation of the detected photon energy may have the impact point of $\vec{p}_{\text {miss }}$ in the LKr sensitive region, whereas the missing photon truly points to the LAV system. Imposing a lower threshold to the missing energy mitigates these effects, as shown by MC simulation. The missing energy evaluated from the energies of the kaon, pion, and photon LKr cluster, $E_{\text {miss }}=E_{K}-E_{\pi}-E_{\gamma}$, is required to be at least $5 \mathrm{GeV}$ above its kinematic lower limit, calculated for the decay of a $\pi^{0}$ to a photon and a particle of mass squared $M_{\text {miss }}^{2}$.

- No in-time NA48-CHOD candidates must be found except for those geometrically associated with the $\pi^{+}$. This condition is referred to as the NA48-CHOD Extraactivity cut.

A total of 8915 events satisfy these criteria.

\subsection{Background evaluation}

After signal selection, MC studies suggest that all background events are $K^{+} \rightarrow \pi^{+} \pi^{0}(\gamma)$ decays, in which one of the photons from the decay $\pi^{0} \rightarrow \gamma \gamma$ is lost due to photo-nuclear interactions or conversions downstream of the NA48-CHOD hodoscope. The detected photon might be correctly reconstructed or its energy might be systematically underestimated due to conversion downstream of the NA48-CHOD hodoscope (e.g. in the LKr cryostat). Background channels $K^{+} \rightarrow \pi^{0} e^{+} \nu_{e}, K^{+} \rightarrow \pi^{0} \mu^{+} \nu_{\mu}, K^{+} \rightarrow \pi^{+} \pi^{0} \gamma(\mathrm{DE}), K^{+} \rightarrow$ $\pi^{+} \pi^{0} \gamma(\mathrm{INT}), K^{+} \rightarrow \mu^{+} \nu_{\mu}(\gamma), K^{+} \rightarrow e^{+} \nu_{e}(\gamma)$ and $K^{+} \rightarrow \pi^{+} \pi^{0} \pi^{0}$ are expected to yield less than one selected event (DE refers to the direct emission component, while INT refers to the interference between DE and IB amplitudes).

To evaluate the expected background, a data-driven approach is used. The data selection of section 3.2 is applied but the NA48-CHOD Extra activity cut is partially inverted: events with one in-time NA48-CHOD candidate geometrically associated with the detected photon are rejected, while the presence of candidates far from both the $\pi^{+}$and photon impact points to the NA48-CHOD hodoscope is required. This allows the selection of a data control sample of $\pi^{0} \rightarrow \gamma \gamma$ events with one photon lost because of conversion upstream of the NA48-CHOD. Ensuring the presence of a second photon with no overlap with the signal sample, the control sample can be used to evaluate the expected $M_{\text {miss }}^{2}$ background distribution with a bias that is below the statistical uncertainty, as verified by MC simulations. The control sample is scaled to the signal sample in a side-band region adjacent to but not overlapping with the $A^{\prime}$ search region. Background considerations suggest considering a minimum mass of $30 \mathrm{MeV} / c^{2}$ for the $A^{\prime}$ search. Similarly, acceptance and yield considerations suggest considering a maximum mass of $130 \mathrm{MeV} / c^{2}$. Given the expected mass resolution 

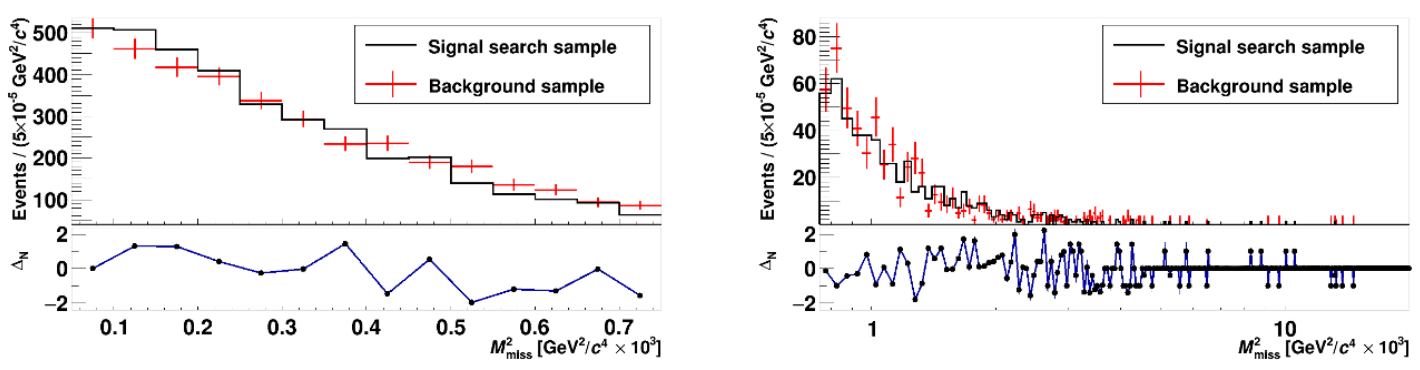

Figure 3. $M_{\text {miss }}^{2}$ distribution of samples for $A^{\prime}$ search (black) and background (red, with error bars). The scaling factors are evaluated in the region shown in the left panel. The search region is shown in the right panel. In the bottom panels, the difference $\Delta_{N}$ between the two $M_{\text {miss }}^{2}$ spectra in units of its standard deviation is shown.

discussed in the next section, the search region is $0.00075<M_{\text {miss }}^{2}<0.01765 \mathrm{GeV}^{2} / c^{4}$. The scaling window used corresponds to $0.00005<M_{\text {miss }}^{2}<0.00075 \mathrm{GeV}^{2} / c^{4}$ (figure 3 , left).

Particular care has been taken to avoid a possible trigger-induced bias when evaluating the expected background. The signal trigger applies the $Q X$-veto condition, rejecting events with in-time signals in opposite CHOD quadrants. To account for the $Q X$-veto potential inefficiency, signal-selected and background samples are divided according to whether the impact points of $\vec{p}_{\text {miss }}$ and of the charged pion track lie in opposite CHOD quadrants or not. The uncertainty on the scale factors is included in the evaluation of the upper limit. The distributions of $M_{\text {miss }}^{2}$ for the signal search and the scaled background samples are shown in figure 3, right.

\section{Search for an $A^{\prime}$ signal}

The expected $M_{\text {miss }}^{2}$ distribution for an $A^{\prime}$ signal and the selection efficiency are evaluated using MC simulations of the $\pi^{0} \rightarrow A^{\prime} \gamma$ decay, with $M_{A^{\prime}}$ ranging from 30 to $130 \mathrm{MeV} / c^{2}$ in steps of $10 \mathrm{MeV} / c^{2}$.

Given the expected background, for each $A^{\prime}$ mass value the signal region optimizing the upper limit in a background-only hypothesis is defined as a $\pm 1 \sigma_{M_{\text {miss }}^{2}}$ window around the expected $M_{\text {miss }}^{2}$ peak value, where $\sigma_{M_{\text {miss }}^{2}}$ is the resolution. The resolution slowly degrades with increasing $M_{A^{\prime}}$ (figure 4). This behaviour is dominated by the relative resolution on the photon energy measured with the LKr calorimeter: the higher the $A^{\prime}$ mass, the lower the energy of the detected photon. The dependence of the resolution on the mass is parameterized with a polynomial function to allow interpolation in the whole search region.

To determine the reliability of the simulation of the missing mass resolution, data and MC simulation are compared for fully reconstructed $K^{+} \rightarrow \pi^{+} \pi^{0}, \pi^{0} \rightarrow \gamma e^{+} e^{-}$decays. The resolution on $\left(P_{K}-P_{\pi}-P_{\gamma}\right)^{2}-\left(P_{e^{+}}+P_{e^{-}}\right)^{2}$ is studied as a function of the di-lepton mass $\left(P_{e^{+}}\right.$and $P_{e^{-}}$are the positron and electron 4 -momenta). Data and MC resolutions are found to agree within $10 \%$. The uncertainty on the resolution is considered in the evaluation of the systematic error on the observed limit. 


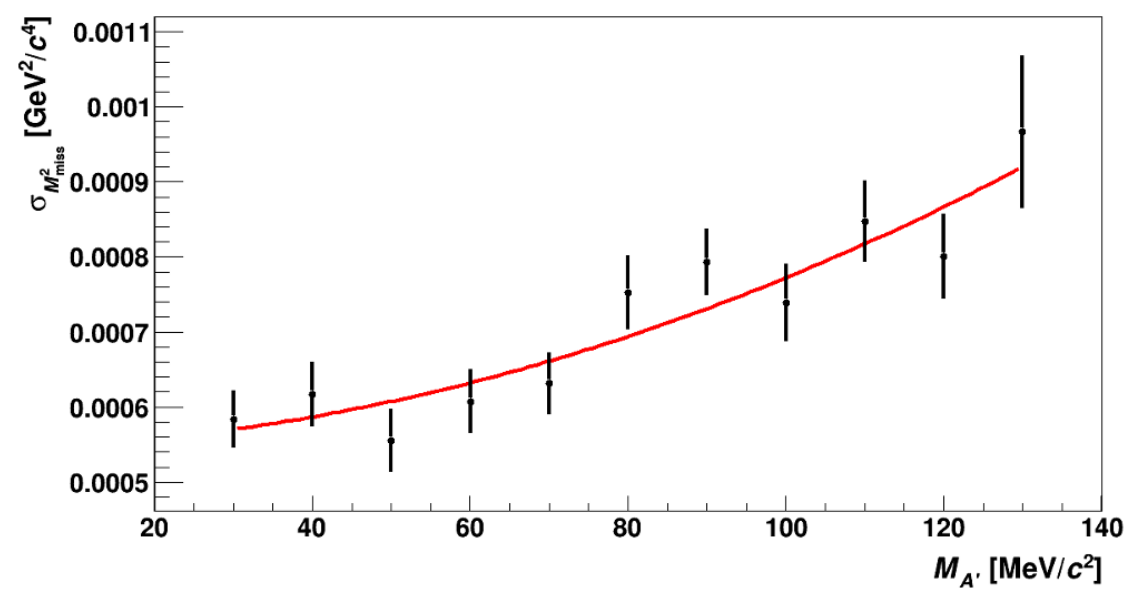

Figure 4. Resolution on the squared missing mass for events satisfying the signal selection criteria, from the simulation of the decay chain $K^{+} \rightarrow \pi^{+} \pi^{0}$ followed by $\pi^{0} \rightarrow A^{\prime} \gamma$, as a function of $M_{A^{\prime}}$. A polynomial function describing the mass dependence is also displayed.

\subsection{Efficiency corrections}

The selection efficiency, $\varepsilon_{\text {sel }}$, is evaluated by MC simulation. A study of the signal loss due to effects not included or not reliably modeled in the MC simulation has been performed. The signal selection requires the presence of a single photon in the final state. Therefore, an efficiency loss is expected due to in-time accidental activity from upstream decays of kaons and pions in the beam, or to the decay $K^{+} \rightarrow \pi^{+} \pi^{0} \gamma$ if the radiative photon is sufficiently hard to be detected. The expected contribution from the former source, the so-called random-veto effect, is evaluated with data collected with the control trigger: information from out-of-time windows from various data samples of $K^{+} \rightarrow \pi^{+} \pi^{0}, \pi^{0} \rightarrow \gamma \gamma$ decays is used for this purpose. For the latter source, MC simulations of the radiative decay are combined with the measured photon detection efficiency. The overall loss due to the two effects is $\left(19.7 \pm 0.2_{\text {stat }} \pm 1.5_{\text {syst }}\right) \%$, dominated by the random-veto contribution. The systematic error includes an estimate of the reliability of the control samples used to reproduce the random-veto effect for the signal sample (0.7\%) and a conservative evaluation of the uncertainty on the detection efficiency of the radiative photon (1.3\%).

The trigger efficiency, $\varepsilon_{\operatorname{trg}}$, is evaluated using an MC simulation with data inputs. Data samples of $K^{+} \rightarrow \pi^{+} \pi^{0}$ events are selected from the control trigger in which exactly one photon LKr cluster is present. The missing momentum $\vec{p}_{\text {miss }}$ must point towards one of the LAV stations, thus ensuring the absence of the second photon in the LKr calorimeter. To mimic the signal sample, in which the $Q X$-veto condition is applied, this control sample is reduced to geometrical configurations in which the selected photon and the charged pion do not traverse opposite CHOD quadrants. The signal-trigger efficiency is obtained as the fraction of events satisfying the signal-trigger chain. The efficiency is binned as a function of the total energy released in the LKr calorimeter. To reproduce the trigger condition, which requires the total energy release to be below $20 \mathrm{GeV}$, the binned efficiency is used as an event-weight for the MC simulation of the signal. The expected distribution of the total 


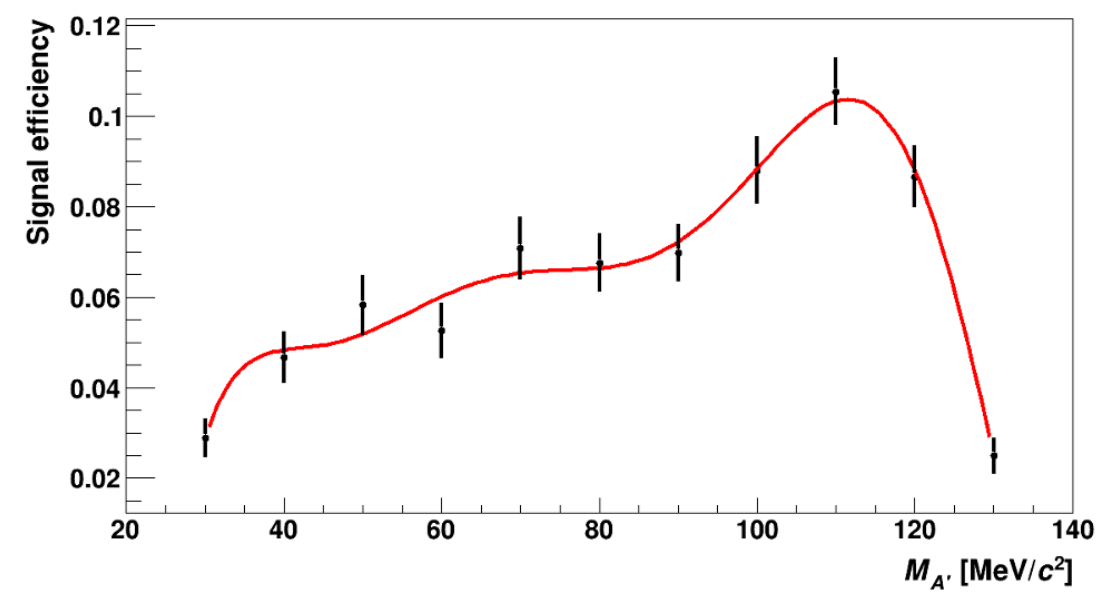

Figure 5. Total efficiency as a function of $M_{A^{\prime}}$. A polynomial function is used to interpolate the global efficiency in the range $30<M_{A^{\prime}}<130 \mathrm{MeV} / c^{2}$.

LKr energy for the signal is therefore convoluted with the data-measured trigger efficiency. The inefficiency induced by the L1 is determined with data-driven methods and is found to be less than $3 \%$.

The total efficiency combining $\varepsilon_{\mathrm{sel}}, \varepsilon_{\mathrm{trg}}$, and the mass-window acceptance $\varepsilon_{\text {mass }}$ determined by $\mathrm{MC}$ simulation is shown as a function of $M_{A^{\prime}}$ in figure 5. It is parameterized with a polynomial function to interpolate in the range $30 \mathrm{MeV} / c^{2}<M_{A^{\prime}}<130 \mathrm{MeV} / c^{2}$. The dependence of the efficiency on $M_{A^{\prime}}$ is dominated by kinematic effects: a heavy $A^{\prime}$ is emitted collinear to a soft visible photon, thus losses can occur both due to the photon detection efficiency and to the isolation cut.

\subsection{Evaluation of the upper limit}

The observed data and the expected background counts are evaluated by integrating the corresponding $M_{\text {miss }}^{2}$ spectrum (figure 3, right) in a $\pm 1 \sigma_{M_{\text {miss }}^{2}}$ signal search window. To avoid the case of exactly zero expected counts, background events lying above $0.005 \mathrm{GeV}^{2} / c^{4}$ ("flat region") are grouped into a single bin. For the signal windows that overlap the flat region, the background entries in the single bin are scaled by the ratio of the signal window width to the flat region width and the errors are evaluated accordingly.

Using the $\mathrm{CL}_{\mathrm{s}}$ algorithm [5], frequentist $90 \%$ confidence intervals are determined for the number of signal events. The upper limits are compatible within two standard deviations with the fluctuation expected in a background-only scenario.

The $90 \%$ CL upper limits obtained on the coupling parameter $\epsilon^{2}$ as a function of $M_{A^{\prime}}$ are shown in figure 6 . The limit from the number of observed events (solid curve) is compared to the bands with $68 \%$ and $95 \%$ coverage in the absence of signal: no statistically significant excess is detected. 


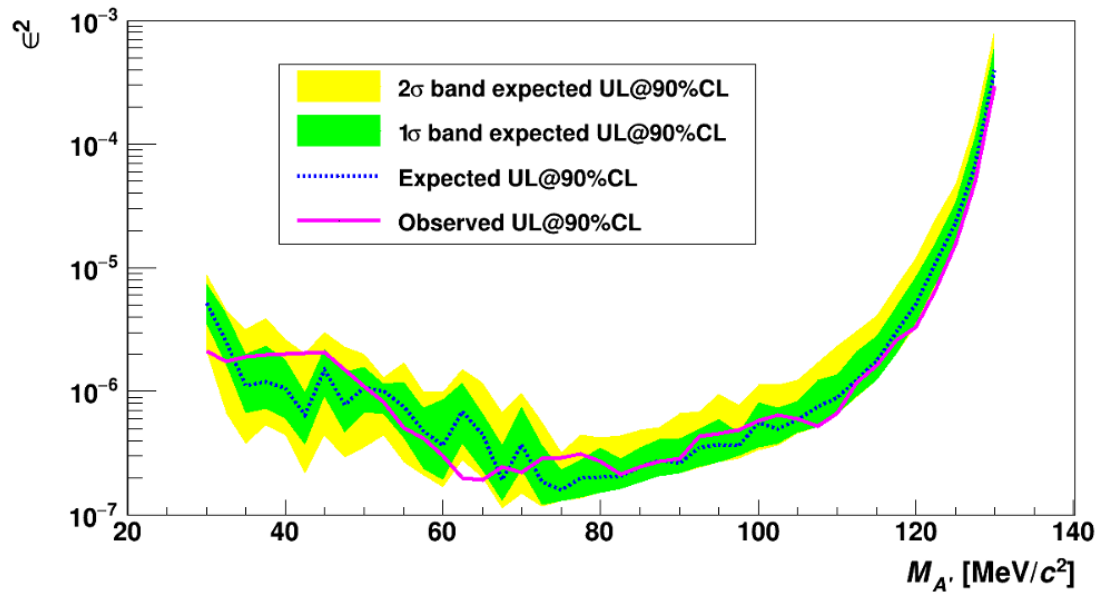

Figure 6. Upper limits at $90 \% \mathrm{CL}$ on the dark photon coupling strength $\left(\epsilon^{2}\right)$ as a function of the mass $\left(M_{A^{\prime}}\right)$. The limit obtained from data (solid line) should be compared to that expected in the absence of signal: the median of the upper-limit distribution in the background-only hypothesis is shown by the dashed line and the corresponding fluctuation bands with $68 \%$ and $95 \%$ coverage are shown by the shaded areas.

\subsection{Systematic errors}

Various parameters used in the statistical procedure have been varied to evaluate the systematic uncertainty on the calculated upper limits. The lower edge of the window used to evaluate the scale factors to compare background and signal-search samples has been varied using the following additional values: $-0.00015,0.00015,0.00025 \mathrm{GeV}^{2} / c^{4}$. The first value implies using the peak of the background distribution for scaling, while the other values correspond to using smaller and smaller portions towards positive values of the $M_{\text {miss }}^{2}$ distribution. For each of these choices, the scaling has been re-evaluated and an upper limit has been obtained. The signal window has been varied to $\pm 0.9, \pm 1.1$, and $\pm 2 \sigma_{M_{\text {miss }}^{2}}$. The extent of the flat region has been varied by moving its lower edge to 0.004 and $0.006 \mathrm{GeV}^{2} / c^{4}$ : these two values correspond to a variation larger than one standard deviation of the signal distribution.

The uncertainties on the signal efficiency, including statistical and systematic errors, have been considered in the evaluation of the upper limit. A confidence band has been calculated for the polynomial interpolation based on the ten efficiency points of figure 5: each interpolated value is taken with the total relative uncertainty of the nearest efficiency point. Moreover, for each efficiency point, the results obtained using the polynomial interpolation have been compared to those using the central values.

No significant deviation beyond the statistical uncertainty has been observed in these studies.

To prove the discovery sensitivity of the analysis, a dark photon signal is injected into the data and the statistical treatment is applied to this altered sample. The $M_{\text {miss }}^{2}$ spectrum corresponding to the MC simulation of an $A^{\prime}$ with $80 \mathrm{MeV} / c^{2}$ mass is scaled according to four different values of the coupling strength $\epsilon^{2}: 6.4 \times 10^{-7}, 10^{-6}$, and $4 \times 10^{-6}$. The scale 
factor applied to each $A^{\prime}$ signal takes into account the full selection and trigger efficiency with its uncertainty. The scaled histograms are added to the data distribution.

The upper limits for these altered samples demonstrate that the method described in this work is able to detect such $A^{\prime}$ signals: for all of the above $\epsilon^{2}$ values, the upper limits found exceed the limit from the background-only hypothesis beyond its $95 \%$ coverage.

\section{Search for the $\pi^{0} \rightarrow \gamma \nu \bar{\nu}$ decay}

With slight modifications to the analysis, a search has been conducted for the decay $\pi^{0} \rightarrow$ $\gamma \nu \bar{\nu}$, for which the BR is expected to be of the order of $10^{-18}[6]$ within the SM. The present experimental limit is $\operatorname{BR}\left(\pi^{0} \rightarrow \gamma \nu \bar{\nu}\right)<6 \times 10^{-4}$ at $90 \%$ CL [7]. The strategy to search for this decay is the same as that used for the $A^{\prime}$, based on the comparison of data and expected background counts in a given $M_{\text {miss }}^{2}$ interval. A MC simulation of the decay is performed using the phase-space density in [6]. The combined efficiency for the signal selection of section 3.2 and the trigger conditions is $(14.0 \pm 0.6) \%$. The range $0.0054 \mathrm{GeV}^{2} / c^{4}<M_{\text {miss }}^{2}<M_{\pi^{0}}^{2}$ is used as the signal-search window after MC optimization of the expected limit in the background-only hypothesis. The $M_{\text {miss }}^{2}$ window chosen contains $(52.8 \pm 1.7) \%$ of the signal events. The number of observed events is 7 , while the expected background counts are $12 \pm 4$. The $\mathrm{CL}_{\mathrm{s}}$ technique provides an upper limit on the number $N_{s}$ of decays observed, $N_{s}<5.6$, which is compatible (within one standard deviation) with the results from the expected background fluctuations. After applying the efficiency corrections, the $90 \%$ CL upper limit obtained is:

$$
\operatorname{BR}\left(\pi^{0} \rightarrow \gamma \nu \bar{\nu}\right)<1.9 \times 10^{-7} .
$$

\section{Conclusions}

A search for an invisible dark photon $A^{\prime}$ has been performed, exploiting the efficient photonveto capability and high resolution tracking of the NA62 detector. The signal stems from the chain $K^{+} \rightarrow \pi^{+} \pi^{0}$ followed by $\pi^{0} \rightarrow A^{\prime} \gamma$. Given the kaon, charged pion, and photon 4-momenta, the squared missing mass $M_{\text {miss }}^{2}=\left(P_{K}-P_{\pi}-P_{\gamma}\right)^{2}$ is expected to peak at the squared $A^{\prime}$ mass for the signal and at zero for the dominant background, $\pi^{0} \rightarrow \gamma \gamma$ decays with one photon undetected. A peak search has been conducted, comparing signal-selected samples and data-driven background estimates. Using the $\mathrm{CL}_{\mathrm{s}}$ method, no significant statistical excess has been identified and upper limits on the coupling strength $\epsilon^{2}$ in the mass range $30-130 \mathrm{MeV} / c^{2}$ have been set, improving on the previous limits over the mass range $60-110 \mathrm{MeV} / c^{2}$ (figure 7 ).

It should be noted that the experimental technique used here differs from that of previous results. At BaBar, positron-electron annihilations to one photon and one dark photon at the centre of mass energy of the $\Upsilon$ resonances should produce energetic singlephoton events [8]. At NA64, dark photons produced by a $100 \mathrm{GeV}$ electron beam dumped into a calorimeter are supposed to yield an excess of events with large missing energy [9]. As a consequence, models different from that of eq. (1.1) and e.g. involving suppressed darkphoton lepton couplings [10], might produce a signal at NA62 notwithstanding the NA64 


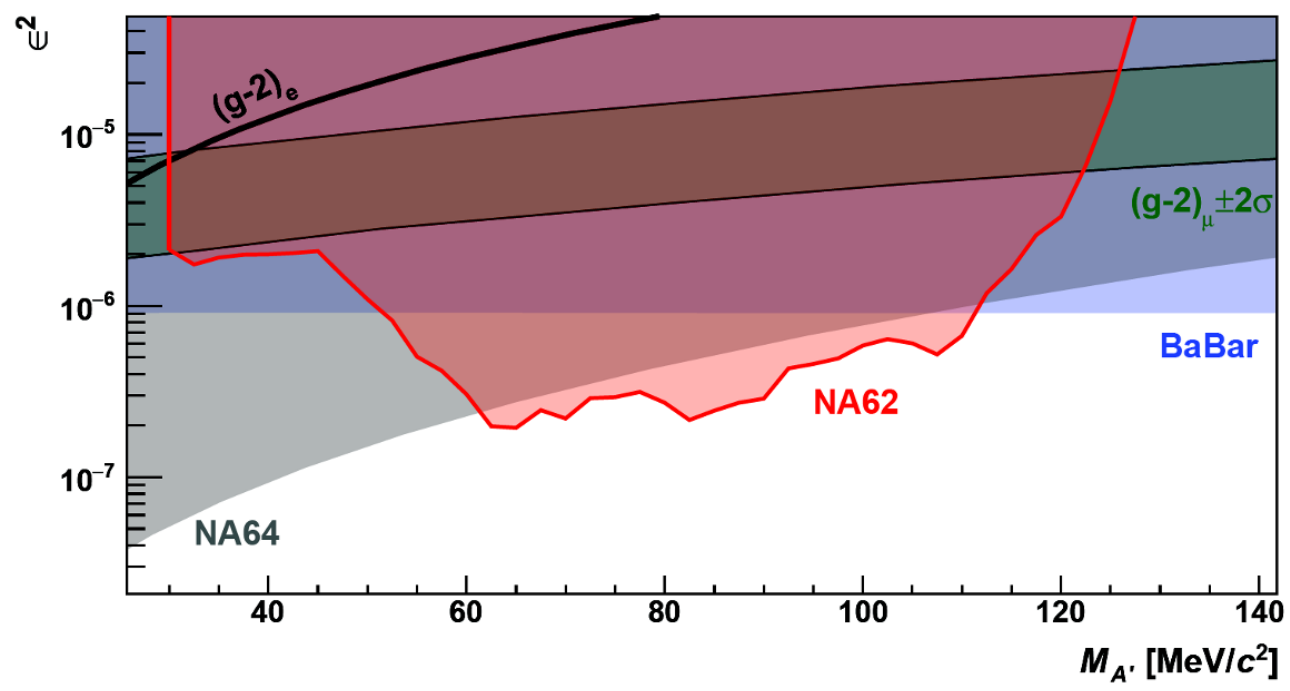

Figure 7. Upper limit at $90 \%$ CL from NA62 (red region) in the $\epsilon^{2}$ vs $M_{A^{\prime}}$ plane with $A^{\prime}$ decaying into invisible final states. The limits from the BaBar [8] (blue) and NA64 [9] (light grey) experiments are shown. The green band shows the region of the parameter space corresponding to an explanation of the discrepancy between the measured [11] and expected values of the anomalous muon magnetic moment $(g-2)_{\mu}[12]$ in terms of a contribution from the $A^{\prime}$ in the quantum loops [13, 14]. The region above the black line is excluded by the agreement of the anomalous magnetic moment of the electron $(g-2)_{e}$ with its expected value [15-17].

and BaBar experimental results. The measurement of the BR for the decay $K^{+} \rightarrow \pi^{+} \nu \bar{\nu}$ by the E787 and E949 experiments [18] can be interpreted as a limit on the BR for the decay $K^{+} \rightarrow \pi^{+} A^{\prime}$ as a function of the $A^{\prime}$ mass. However, this interpretation is modeldependent: if a mixing of the dark photon to the $Z$ boson is introduced, the lower edge of the exclusion bound increases by a factor of 7 [19]. In the most conservative scenario, not shown in figure 7, the upper limit from E787-E949 partially overlaps with the $(g-2)_{\mu}$ band in the mass ranges $83-113$ and $176-243 \mathrm{MeV} / c^{2}$.

Finally, an upper limit has been set for the branching ratio of the decay $\pi^{0} \rightarrow \gamma \nu \bar{\nu}$, $\mathrm{BR}<1.9 \times 10^{-7}$ at $90 \% \mathrm{CL}$, improving the current limit by more than three orders of magnitude.

\section{Acknowledgments}

It is a pleasure to express our appreciation to the staff of the CERN laboratory and the technical staff of the participating laboratories and universities for their efforts in the operation of the experiment and data processing.

The cost of the experiment and of its auxiliary systems were supported by the funding agencies of the Collaboration Institutes. We are particularly indebted to: F.R.S.FNRS (Fonds de la Recherche Scientifique - FNRS), Belgium; BMES (Ministry of Education, Youth and Science), Bulgaria; NSERC (Natural Sciences and Engineering Research Council), Canada; NRC (National Research Council) contribution to TRIUMF, Canada; MEYS (Ministry of Education, Youth and Sports), Czech Republic; BMBF 
(Bundesministerium für Bildung und Forschung) contracts 05H12UM5, 05H15UMCNA and 05H18UMCNA, Germany; INFN (Istituto Nazionale di Fisica Nucleare), Italy; MIUR (Ministero dell'Istruzione, dell'Università e della Ricerca), Italy; CONACyT (Consejo Nacional de Ciencia y Tecnología), Mexico; IFA (Institute of Atomic Physics), Romania; INR-RAS (Institute for Nuclear Research of the Russian Academy of Sciences), Moscow, Russia; JINR (Joint Institute for Nuclear Research), Dubna, Russia; NRC (National Research Center) "Kurchatov Institute" and MESRF (Ministry of Education and Science of the Russian Federation), Russia; MESRS (Ministry of Education, Science, Research and Sport), Slovakia; CERN (European Organization for Nuclear Research), Switzerland; STFC (Science and Technology Facilities Council), United Kingdom; NSF (National Science Foundation) Award Number 1506088, U.S.A.; ERC (European Research Council) "UniversaLepto" advanced grant 268062, "KaonLepton" starting grant 336581, Europe.

Individuals have received support from: Charles University (project GA UK number 404716), Czech Republic; Ministry of Education, Universities and Research (MIUR "Futuro in ricerca 2012" grant RBFR12JF2Z, Project GAP), Italy; Russian Foundation for Basic Research (RFBR grants 18-32-00072, 18-32-00245), Russia; the Royal Society (grants UF100308, UF0758946), United Kingdom; STFC (Rutherford fellowships ST/J00412X/1, ST/M005798/1), United Kingdom; ERC (grants 268062, 336581 and starting grant 802836 "AxScale").

Open Access. This article is distributed under the terms of the Creative Commons Attribution License (CC-BY 4.0), which permits any use, distribution and reproduction in any medium, provided the original author(s) and source are credited.

\section{References}

[1] L.B. Okun, Limits of electrodynamics: paraphotons?, Sov. Phys. JETP 56 (1982) 502 [INSPIRE].

[2] B. Holdom, Two U(1)'s and Epsilon Charge Shifts, Phys. Lett. B 166 (1986) 196 [inSPIRE].

[3] NA62 collaboration, The beam and detector of the NA62 experiment at CERN, 2017 JINST 12 P05025 [arXiv:1703.08501].

[4] NA62 collaboration, First search for $K^{+} \rightarrow \pi^{+} \nu \bar{\nu}$ using the decay-in-flight technique, Phys. Lett. B 791 (2019) 156 [arXiv:1811.08508] [INSPIRE].

[5] A.L. Read, Presentation of search results: The CL(s) technique, J. Phys. G 28 (2002) 2693 [INSPIRE].

[6] L. Arnellos, W.J. Marciano and Z. Parsa, The Decay $\pi^{0} \rightarrow \nu \bar{\nu} \gamma$, Nucl. Phys. B 196 (1982) 365 [INSPIRE].

[7] M.S. Atiya et al., Search for the decay $\pi^{0} \rightarrow \gamma+X$, Phys. Rev. Lett. 69 (1992) 733 [INSPIRE].

[8] BABAR collaboration, Search for Invisible Decays of a Dark Photon Produced in $e^{+} e^{-}$ Collisions at BaBar, Phys. Rev. Lett. 119 (2017) 131804 [arXiv:1702.03327] [INSPIRE].

[9] NA64 collaboration, Search for vector mediator of Dark Matter production in invisible decay mode, Phys. Rev. D 97 (2018) 072002 [arXiv:1710.00971] [INSPIRE]. 
[10] P. Ilten, Y. Soreq, M. Williams and W. Xue, Serendipity in dark photon searches, JHEP 06 (2018) 004 [arXiv : 1801.04847] [INSPIRE].

[11] Muon G-2 collaboration, Final Report of the Muon E821 Anomalous Magnetic Moment Measurement at BNL, Phys. Rev. D 73 (2006) 072003 [hep-ex/0602035] [INSPIRE].

[12] Particle Data Group collaboration, Review of Particle Physics, Phys. Rev. D 98 (2018) 030001 [INSPIRE].

[13] P. Fayet, U-boson production in $e^{+} e^{-}$annihilations, psi and Upsilon decays and Light Dark Matter, Phys. Rev. D 75 (2007) 115017 [hep-ph/0702176] [InSPIRE].

[14] M. Pospelov, Secluded U(1) below the weak scale, Phys. Rev. D 80 (2009) 095002 [arXiv:0811.1030] [INSPIRE].

[15] D. Hanneke, S. Fogwell and G. Gabrielse, New Measurement of the Electron Magnetic Moment and the Fine Structure Constant, Phys. Rev. Lett. 100 (2008) 120801 [arXiv:0801.1134] [INSPIRE].

[16] R. Bouchendira, P. Clade, S. Guellati-Khelifa, F. Nez and F. Biraben, New determination of the fine structure constant and test of the quantum electrodynamics, Phys. Rev. Lett. 106 (2011) 080801 [arXiv: 1012.3627] [INSPIRE].

[17] T. Aoyama, M. Hayakawa, T. Kinoshita and M. Nio, Tenth-Order QED Contribution to the Electron g-2 and an Improved Value of the Fine Structure Constant, Phys. Rev. Lett. 109 (2012) 111807 [arXiv:1205.5368] [INSPIRE].

[18] BNL-E949 collaboration, Study of the decay $K^{+} \rightarrow \pi^{+} \nu \bar{\nu}$ in the momentum region $140<P_{\pi}<199 \mathrm{MeV} / c$, Phys. Rev. D 79 (2009) 092004 [arXiv:0903.0030] [InSPIRE].

[19] H. Davoudiasl, H.-S. Lee and W.J. Marciano, Muon g - 2, rare kaon decays and parity violation from dark bosons, Phys. Rev. D 89 (2014) 095006 [arXiv: 1402.3620] [INSPIRE]. 
The NA62 collaboration

Université Catholique de Louvain, Louvain-La-Neuve, Belgium

E. Cortina Gil, A. Kleimenova, E. Minucci ${ }^{1}$, S. Padolski ${ }^{2}$, P. Petrov, A. Shaikhiev ${ }^{3}$, R. Volpe

Faculty of Physics, University of Sofia, Sofia, Bulgaria

G. Georgiev ${ }^{4}$, V. Kozhuharov ${ }^{4}$, L. Litov

TRIUMF, Vancouver, British Columbia, Canada

T. Numao, Y. Petrov, B. Velghe

University of British Columbia, Vancouver, British Columbia, Canada

D. Bryman, J. Fu ${ }^{5}$

Charles University, Prague, Czech Republic

T. Husek ${ }^{6}$, J. Jerhot, K. Kampf, M. Zamkovsky

Institut für Physik and PRISMA Cluster of excellence, Universität Mainz, Mainz, Germany

R. Aliberti, G. Khoriauli ${ }^{7}$, J. Kunze, D. Lomidze ${ }^{8}$, L. Peruzzo, M. Vormstein, R. Wanke

Dipartimento di Fisica e Scienze della Terra dell'Università e INFN, Sezione di Ferrara, Ferrara, Italy

P. Dalpiaz, M. Fiorini, I. Neri, A. Norton, F. Petrucci, H. Wahl

INFN, Sezione di Ferrara, Ferrara, Italy

A. Cotta Ramusino, A. Gianoli

Dipartimento di Fisica e Astronomia dell'Università e INFN, Sezione di Firenze, Sesto Fiorentino, Italy

E. Iacopini, G. Latino, M. Lenti, A. Parenti

INFN, Sezione di Firenze, Sesto Fiorentino, Italy

A. Bizzeti ${ }^{9}$, F. Bucci

Laboratori Nazionali di Frascati, Frascati, Italy

A. Antonelli, G. Lanfranchi, G. Mannocchi, S. Martellotti, M. Moulson, T. Spadaro*

Dipartimento di Fisica "Ettore Pancini" e INFN, Sezione di Napoli, Napoli, Italy

F. Ambrosino, T. Capussela, M. Corvino, D. Di Filippo, P. Massarotti, M. Mirra*, M. Napolitano, G. Saracino

Dipartimento di Fisica e Geologia dell'Università e INFN, Sezione di Perugia, Perugia, Italy

G. Anzivino, F. Brizioli, E. Imbergamo, R. Lollini, R. Piandani, C. Santoni

INFN, Sezione di Perugia, Perugia, Italy

M. Barbanera ${ }^{10}$, P. Cenci, B. Checcucci, P. Lubrano, M. Lupi ${ }^{11}$, M. Pepe, M. Piccini 
Dipartimento di Fisica dell'Università e INFN, Sezione di Pisa, Pisa, Italy

F. Costantini, L. Di Lella, N. Doble, M. Giorgi, S. Giudici, G. Lamanna, E. Lari, E. Pedreschi, M. Sozzi

INFN, Sezione di Pisa, Pisa, Italy

C. Cerri, R. Fantechi, L. Pontisso, F. Spinella

Scuola Normale Superiore e INFN, Sezione di Pisa, Pisa, Italy

I. Mannelli

Dipartimento di Fisica, Sapienza Università di Roma e INFN, Sezione di Roma I, Roma, Italy

G. D'Agostini, M. Raggi

INFN, Sezione di Roma I, Roma, Italy

A. Biagioni, E. Leonardi, A. Lonardo, P. Valente, P. Vicini

INFN, Sezione di Roma Tor Vergata, Roma, Italy

R. Ammendola, V. Bonaiuto ${ }^{12}$, A. Fucci, A. Salamon, F. Sargeni ${ }^{13}$

Dipartimento di Fisica dell'Università e INFN, Sezione di Torino, Torino, Italy R. Arcidiacono ${ }^{14}$, B. Bloch-Devaux, M. Boretto ${ }^{15}$, E. Menichetti, E. Migliore, D. Soldi

INFN, Sezione di Torino, Torino, Italy

C. Biino, A. Filippi, F. Marchetto

Instituto de Física, Universidad Autónoma de San Luis Potosí, San Luis Potosí, Mexico

J. Engelfried, N. Estrada-Tristan ${ }^{16}$

Horia Hulubei national Institute of Physics and Nuclear Engineering, Bucharest-Magurele, Romania

A. M. Bragadireanu, S. A. Ghinescu, O. E. Hutanu

Joint Institute for Nuclear Research, Dubna, Russia

T. Enik, V. Falaleev, V. Kekelidze, A. Korotkova, D. Madigozhin, M. Misheva ${ }^{17}$,

N. Molokanova, S. Movchan, I. Polenkevich, Yu. Potrebenikov, S. Shkarovskiy,

A. Zinchenko ${ }^{\dagger}$

Institute for Nuclear Research of the Russian Academy of Sciences, Moscow, Russia

S. Fedotov, E. Gushchin, A. Khotyantsev, Y. Kudenko ${ }^{18}$, V. Kurochka, M. Medvedeva, A. Mefodev

Institute for High Energy Physics - State Research Center of Russian Federation, Protvino, Russia

S. Kholodenko, V. Kurshetsov, V. Obraztsov, A. Ostankov, V. Semenov ${ }^{\dagger}$, V. Sugonyaev, O. Yushchenko 
Faculty of Mathematics, Physics and Informatics, Comenius University, Bratislava, Slovakia

L. Bician, T. Blazek, V. Cerny, Z. Kucerova

\section{CERN, European Organization for Nuclear Research, Geneva, Switzerland}

J. Bernhard, A. Ceccucci, H. Danielsson, N. De Simone ${ }^{19}$, F. Duval, B. Döbrich, L. Federici,

E. Gamberini, L. Gatignon, R. Guida, F. Hahn ${ }^{\dagger}$, E. B. Holzer, B. Jenninger, M. Koval,

P. Laycock ${ }^{2}$, G. Lehmann Miotto, P. Lichard, A. Mapelli, R. Marchevski, K. Massri,

M. Noy, V. Palladino ${ }^{20}$, M. Perrin-Terrin ${ }^{21,22}$, J. Pinzino, V. Ryjov, S. Schuchmann, S. Venditti

\section{University of Birmingham, Birmingham, United Kingdom}

T. Bache, M. B. Brunetti, V. Duk, V. Fascianelli23 , J. R. Fry, F. Gonnella, E. Goudzovski, L. Iacobuzio, C. Lazzeroni, N. Lurkin, F. Newson, C. Parkinson, A. Romano, A. Sergi, A. Sturgess, J. Swallow

\section{University of Bristol, Bristol, United Kingdom}

H. Heath, R. Page, S. Trilov

University of Glasgow, Glasgow, United Kingdom

B. Angelucci, D. Britton, C. Graham, D. Protopopescu

University of Lancaster, Lancaster, United Kingdom

J. Carmignani, J. B. Dainton, R. W. L. Jones, G. Ruggiero

University of Liverpool, Liverpool, United Kingdom

L. Fulton, D. Hutchcroft, E. Maurice ${ }^{24}$, B. Wrona

George Mason University, Fairfax, Virginia, U.S.A.

A. Conovaloff, P. Cooper, D. Coward ${ }^{25}$, P. Rubin

*: Corresponding author

$\dagger$ : Deceased

1: Present address: Laboratori Nazionali di Frascati, I-00044 Frascati, Italy

2: Present address: Brookhaven National Laboratory, Upton, NY 11973, U.S.A.

3: Also at Institute for Nuclear Research of the Russian Academy of Sciences, 117312 Moscow, Russia

4: Also at Laboratori Nazionali di Frascati, I-00044 Frascati, Italy

5: Present address: UCLA Physics and Biology in Medicine, Los Angeles, CA 90095, U.S.A.

6: Present address: IFIC, Universitat de València - CSIC, E-46071 València, Spain

7: Present address: Universität Würzburg, D-97070 Würzburg, Germany

8: Present address: Universität Hamburg, D-20146 Hamburg, Germany

9: Also at Dipartimento di Fisica, Università di Modena e Reggio Emilia, I-41125 Modena, Italy

10: Present address: INFN, Sezione di Pisa, I-56100 Pisa, Italy

11: Present address: Institut am Fachbereich Informatik und Mathematik, Goethe Universität, D-60323 Frankfurt am Main, Germany

12: Also at Department of Industrial Engineering, University of Roma Tor Vergata, I-00173 Roma, Italy 
13: Also at Department of Electronic Engineering, University of Roma Tor Vergata, I-00173 Roma, Italy

14: Also at Università degli Studi del Piemonte Orientale, I-13100 Vercelli, Italy

15: Also at CERN, European Organization for Nuclear Research, CH-1211 Geneva 23, Switzerland

16: Also at Universidad de Guanajuato, Guanajuato, Mexico

17: Present address: Institute of Nuclear Research and Nuclear Energy of Bulgarian Academy of Science (INRNE-BAS), BG-1784 Sofia, Bulgaria

18: Also at National Research Nuclear University (MEPhI), 115409 Moscow and Moscow Institute of Physics and Technology, 141701 Moscow region, Moscow, Russia

19: Present address: DESY, D-15738 Zeuthen, Germany

20: Present address: Physics Department, Imperial College London, London, SW7 2BW, U.K.

21: Present address: Centre de Physique des Particules de Marseille, Université Aix Marseille, CNRS/IN2P3, F-13288, Marseille, France

22: Also at Université Catholique de Louvain, B-1348 Louvain-La-Neuve, Belgium

23: Present address: Dipartimento di Psicologia, Università di Roma La Sapienza, I-00185 Roma, Italy

24: Present address: Laboratoire Leprince Ringuet, F-91120 Palaiseau, France

25: Also at SLAC National Accelerator Laboratory, Stanford University, Menlo Park, CA 94025, U.S.A. 